\title{
Electronic Absorption Spectral Investigations and Thermodynamic Properties of Urea Maleic Acid for Nonlinear Optical Applications
}

\author{
Xiao-Jing LIU ${ }^{a^{*}}$, Xin SUN ${ }^{b}$, Jing-Hua GUO ${ }^{c}$, Miao-Juan REN ${ }^{d}$
}

School of Physics and Technology, University of Jinan, Jinan 250022, Shandong Province, PR China a631853207@qq.com(Corresponding author email), b1259699347@qq.com, ${ }^{\mathrm{c}} 328758234 @ q q . c o m$, d1193906524@qq.com

${ }^{*}$ Corresponding author

Keywords: Nonlinear Optical Material, DFT, Optical Study.

\begin{abstract}
In this work, theoretical calculations have been performed on urea maleic acid (UMA) by using density functional theory (DFT) method at B3LYP/6-311++G(d,p) basis set levels. In calculation of electronic absorption spectra, TD-DFT calculations have been carried out. The calculated HOMO-LUMO energies confirm the nonlinear optical activity. The correlations between the statistical thermodynamics and temperature have also been obtained. These results discussed in this study will upsurge the knowledge to design and synthesize urea derivatives with exceptional chemical, physical and biological properties.
\end{abstract}

\section{Introduction}

Urea derivatives can be considered as important materials because of their nonlinearities, optical transparency, adequate birefringence and environmental stability. In the present complex one molecule of maleic acid exist in monoionized state, with urea molecule in protonated form (UMA). Previously, experimental techniques has been applied to investigate the crystal grow, thermal, mechanical and defect characteristics of UMA [1-2]. However, to the best of our knowledge, no theoretical studies on the compound of UMA have been available till now. In this study, by density functional theory (DFT) techniques, the optimized geometry, vibrational spectra and assignments, electronic absorption spectra, statistical thermodynamic parameters, molecular electrostatic potential and nonlinear optic (NLO) properties of UMA have been studied. DFT is developing very fast for studying optical and nonlinear optical properties including UV-visible, fluorescence, IR, NMR, Raman spectroscopic properties and polarizabilities of many molecules [3]. The obtained results of this study can shed light on the optical activities of this compound.

\section{Computational Details}

Quantum chemical calculations (QCCs) were carried out for UMA with Gaussian 09W program package [4] using DFT/B3LYP method supplemented with the standard 6-311++G(d,p) basis set (Fig.1). Gaussview program [5] has been considered to visualize the molecular structure, vibrational and electronic absorption spectra. The time dependent density functional theory (TD-DFT) method [6] was used to calculate the electronic absorption spectra. Electronic properties of UMA were also computed. Moreover, the changes in the thermodynamic functions (the enthalpy, entropy and heat capacity) were investigated for the different temperatures from the vibrational frequency calculations of title molecule. 


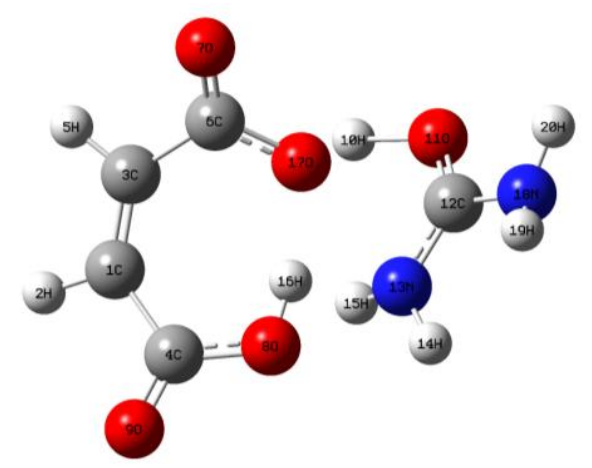

Fig. 1 Optimized geometry of UMA at B3LYP/6-311++G(d,p) level of theory.

\section{Results and Discussion}

\section{TD-DFT Study}

Experimentally, electronic absorption spectrum of the title crystal is showing one bands at $290 \mathrm{~nm}$ as shown in Fig.2(a) and the computed UV spectra predicts one intense electronic transition at $287 \mathrm{~nm}$ Fig.2(b) with an oscillator strength $\mathrm{f}=0.0068$. The visible band could be attributed to high delocalization of $\pi$ - electrons and found close to each other. The FMOs play an important role in the electric and optical properties as well as in UV-Vis spectra and chemical reactions [7]. The HOMO and LUMO energy gap describes the chemical reactivity, kinetic stability, optical polarizability, and chemical softness of a molecule. As our TD-DFT calculations show that electronic absorption energy of $287 \mathrm{~nm}$ corresponds to the transition from the ground state to excited state and is mainly involved one electron excitation from HOMO-1 to LUMO+1 orbitals (Fig.3). It can be seen that the HOMO-1 orbital is localized on urea molecule group while in the LUMO+1 electron are mainly delocalized on maleic acid group.


Fig. 2 (a) Optical absorption spectrum and (b) calculated absorption spectra of UMA

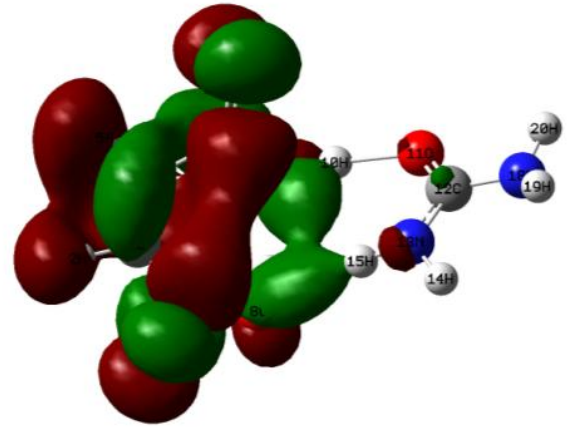

LUMO+1 (-0.418064 eV)

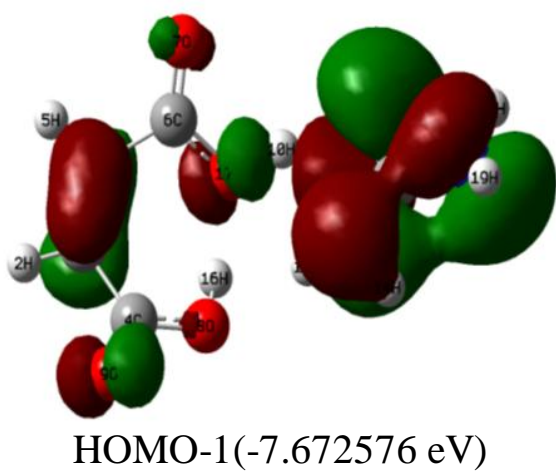

HOMO-1(-7.672576 eV)

Fig. 3 The frontier molecular orbitals (FMOs) of UMA molecule. 


\section{Global Chemical Reactivity Descriptors}

Chemical reactivity indices like chemical hardness $(\eta)$, electronegativity $(\chi)$, electronic chemical potential $(\mu)$, and electrophilicity index $(\omega)$, are calculated using DFT. On the base of frontier molecular orbitals, chemical hardness links to the gap between the HOMO and LUMO and is determined using the equation $\eta=\left(\mathrm{E}_{\mathrm{LUMO}}-\mathrm{E}_{\mathrm{HOMO}}\right) / 2$. Higher the electronegativity of the type which expressed by $\chi=-\left(\mathrm{E}_{\mathrm{HOMO}}+\mathrm{E}_{\mathrm{LUMO}}\right) / 2$, larger is its electron accepting power or rather the electrophilicity. As presented in equation $\mu=\left(\mathrm{E}_{\mathrm{HOMO}}+\mathrm{E}_{\mathrm{LUMO}}\right) / 2$, electronic chemical potential is explained as the negative of electronegativity of a molecule. Global electrophilicity index $(\omega)$, introduced by Parr, is determined using the electronic chemical potential and chemical hardness $\left(\omega=\mu^{2} / 2 \eta\right)$. Electrophilicity index calculates the propensity or ability of a type to accept electrons [8]. It is a measure of the stabilization in energy once a system accepts additional sum of electronic charge from the environment [9].

Tab. 1 The calculated energy values of frontier molecular orbitals (FMOs) and energy values.

\begin{tabular}{lc}
\hline Parameters & Values \\
\hline $\mathrm{E}_{\text {LUMO }}(\mathrm{eV})$ & 36.0979 \\
$\mathrm{E}_{\text {HOMO }}(\mathrm{eV})$ & -7.5273 \\
$\mathrm{E}_{\text {HOMO-ELUMO }}$ gap $(\mathrm{eV})$ & ${ }^{-5.4293}$ \\
Chemical hardness $(\eta)$ & 92 \\
& 96 \\
Chemical potential $(\mu)$ & -4.8146 \\
Electronegativity $(\chi)$ & 32 \\
Electrophilicity index & 32.8126 \\
$(\omega)$ & 34 \\
\hline
\end{tabular}

\section{Non-linear Optical Properties}

The non-linear optical properties of the organic molecules are related to the delocalized $\pi$ electrons that move along molecule. The increase of the conjugation on molecule gives rise to an increase in its non-linear optical properties. One another way to increase non-linear optical properties is to add donor and acceptor groups. If the donor and acceptor groups are powerful, delocalization of $\pi$ electron cloud on organic molecules increases and as a result of this polarizability and first hyperpolarizability of organic molecules increase [10]. Calculated values of electronic dipole moment $(\mu)$, polarizability $(\alpha)$ and the first hyperpolarizability $(\beta)$ are 4.02 Debye, $32.05 \AA^{3}$ and $1.96 \times 10^{-30} \mathrm{~cm}^{5} / \mathrm{esu}$, respectively. These results are greater than those of urea ( $\mu$ and $\beta$ of urea of 1.526 Debye and $0.7803 \times 10^{-30} \mathrm{~cm}^{5} / \mathrm{esu}$ obtained by B3LYP/6-311++G (d, p) method) [11]. These results show that the studied compound exhibit good nonlinear optical activity and can be used as a good non-linear optical material.

\section{Thermodynamic Parameters}

Table 2 shows the thermodynamic parameters are increasing with temperature ranging from 100 to $1000 \mathrm{~K}$ due to the fact that the vibrational intensities increase with the increasing temperature [12]. The correlation equations are as follows and the related fitting factors $\left(\mathrm{R}^{2}\right)$ for these parameters were found to be $0.99829,0.9924$ and 0.99687 , respectively.

$$
\begin{aligned}
& C_{p, m}^{0}=8.3557+0.14654 T-6.84435 \times 10^{-5} T^{2} \quad\left(R^{2}=0.99829\right) \\
& S_{m}^{0}=58.86014+0.20564 T-6.90603 \times 10^{-5} T^{2} \quad\left(R^{2}=0.9924\right)
\end{aligned}
$$




$$
\Delta H_{m}^{0}=-2.93317+0.02868 T+3.55442 \times 10^{-5} T^{2} \quad\left(R^{2}=0.99687\right)
$$

These equations could be used for further studies on the title compound. For example, while studying about the interaction of UMA with another compound, the thermodynamic properties obtained from the above equation can be used to evaluate the change in Gibbs free energy of the reaction, which will in turn comfort to evaluate the spontaneity of the reaction.

Tab. 2 Variation in thermodynamic parameters with temperature for UMA

\begin{tabular}{llll}
\hline Temperature $(\mathrm{K})$ & $C_{p, m}^{0}\left(\mathrm{cal} \mathrm{mol}^{-1} \mathrm{~K}^{-1}\right)$ & $S_{m}^{0}\left(\mathrm{cal} \mathrm{mol}^{-1} \mathrm{~K}^{-1}\right)$ & $\Delta H_{m}^{0}\left(\mathrm{Kcal} \mathrm{mol}^{-1}\right)$ \\
\hline 100 & 21.277 & 75.110 & 1.440 \\
200 & 35.444 & 95.774 & 4.502 \\
298.15 & 45.460 & 117.766 & 6.055 \\
300 & 47.164 & 113.235 & 8.848 \\
400 & 56.933 & 128.758 & 14.267 \\
500 & 64.911 & 142.794 & 20.572 \\
600 & 71.355 & 155.581 & 27.596 \\
700 & 76.580 & 167.293 & 35.200 \\
800 & 80.876 & 178.074 & 43.278 \\
900 & 84.462 & 188.047 & 51.749 \\
1000 & 87.500 & 197.317 & 60.550 \\
\hline
\end{tabular}

\section{Conclusions}

In the current work we have applied quantum chemical techniques to study the electro-optical and nonlinear optical properties of UMA. Based on TD-DFT method, electronic absorption spectra have been calculated, and they are compatible with the experimental ones. The value of dipole moment and first hyperpolarizability of UMA are found to be 2.5 times higher than that of urea. The obtained results reveal that UMA is a good candidate for nonlinear applications having an excellent transparency trade-off value.

\section{Acknowledgement}

This work was supported by the National Natural Science Foundation of China (Grant Nos. 61205175 and 61575081), the Encouragement Foundation for Excellent Middle-aged and Young Scientist of Shandong Province, China (Grant No. BS2011CL008) and the Doctoral Foundation of University of Jinan, China (Grant No. XBS0920).

\section{References}

[1] P. Vinothkumar, R. MohanKumar, R. Jayavel, A.Bhaskaran, Optics \&LaserTechnology 81 (2016) 145-152.

[2] V. Videnova-Adrabinska, J.Mol.Struct. 374 (1996) 199-222.

[3] Mehmet Cinar, Ali Coruh, Mehmet Karabacak, Spectrochim. Acta Part A 122 (2014) 682-689.

[4] M.J. Frisch, et al., Gaussian 09, Revision C. 01, Gaussian Inc., Wallingford CT, 2009.

[5] R. Dennington, T. Keith, J. Millam, Semichem Inc, Shawnee Mission KS, GaussView, Version 5, 2009. 
[6] M.E. Casida, C. Jamorski, K.C. Casida, D.R. Salahub, J. Chem. Phys. 108 (1998) 4439-4449.

[7] I. Fleming, Frontier Orbitals and Organic Chemical Reactions, Wiley, London, 1976.

[8] R.G. Parr, L. Szentpaly, S. Liu, J. Am. Chem. Soc. 121 (1999) 1922-1925.

[9] S. Liu, J. Chem. Sci. 117 (2005) 477-479.

[10] S. Muthu, E.E. Porchelvi, M. Karabacak, A.M. Asiri, S.S. Swathi, J. Mol. Struct. 1081 (2015) 400-412.

[11] Ü. Ceylan, M. Durgun, H. Türkmen, 1089 (2015) 222-232.

[12] H. Tanak, A.A. Agar, O. Büyükgüng€or, J. Mol. Struct. 1048 (2013) 41-50. 